\title{
Effect of Oxygen Exposure on ATP Content of Rat Bronchoalveolar Lavage
}

\author{
WARD R. RICE, MICHAEL BURHANS, AND JONATHAN R. WISPÉ \\ University of Cincinnati Medical Center, Department of Pediatrics, Divisions of Neonatology and Pulmonary \\ Biology, Cincinnati, Ohio 45267-054I
}

\begin{abstract}
Exogenous ATP stimulates surfactant phospholipid secretion in vitro through binding to $\mathrm{P}_{2}$-purinoceptors on the surface of the alveolar type II cell. To determine whether ATP is present in the bronchoalveolar space at concentrations sufficient to stimulate phospholipid secretion, we determined bronchoalveolar lavage ATP content in rats exposed to different inspired oxygen concentrations. Bronchoalveolar lavage ATP content of control animals exposed to room air was $155 \pm 12 \mathrm{nmol} \mathrm{ATP} /$ lavage $(n=17)$ and fell in a dose-dependent fashion to $34 \pm 5$ nmol ATP/lavage in animals exposed to $85 \%$ oxygen for $72 \mathrm{~h}(n=17)$. Airway content of ATP metabolites fell in a similar fashion in response to oxygen exposure. The decrease in airway ATP content after oxygen exposure was also time-dependent. ATP content in young adult male rats exposed to $85 \%$ oxygen decreased from a mean of 236 nmol ATP/lavage on $d 1$ of exposure to $18 \mathrm{nmol} \mathrm{ATP/}$ lavage after $4 \mathrm{~d}$. There was no further decrease in bronchoalveolar lavage ATP content after 4-7 d of oxygen exposure. Despite the fall in airway levels of ATP, disaturated phosphatidylcholine levels increased. Rat bronchoalveolar lavage contained measurable concentrations of ATP, indicating the presence of ATP in the airway at levels which modulate surfactant phospholipid secretion from isolated alveolar type II cells in vitro. Bronchoalveolar lavage ATP content decreased significantly with exposure to oxygen, whereas disaturated phosphatidylcholine content increased. (Pediatr Res 25:396-398, 1989)
\end{abstract}

Abbreviation

DSPC, disaturated phosphatidylcholine

Alveolar type II cells synthesize and secrete surfactant phospholipids in response to a variety of secretagogues which include $\beta$-adrenoceptor agonists (1), phorbol esters (2), calcium ionophores (3), and cytochalasins (4). One of the most potent agonists for surfactant phospholipid secretion in vitro is exogenous ATP, which activates cell surface $\mathrm{P}_{2}$-purinoceptors mobilizing intracellular calcium in association with surfactant phospholipid secretion $(5,6)$. Whether ATP is present in relevant concentrations in the airway and whether ATP plays a similar role in vivo is unknown. We therefore undertook the present study to determine whether ATP is present in bronchoalveolar lavage in concentrations sufficient to mediate surfactant phospholipid secretion in vivo.

Received September 2, 1988; accepted November 22, 1988

Correspondence to Ward R. Rice, M.D., Ph.D., University of Cincinnati Medical Center, Department of Pediatrics, Divisions of Neonatology \& Pulmonary Biology, 231 Bethesda Avenue, Cincinnati, $\mathrm{OH}$ 45267-0541.

Supported by NIH Grants HL38764 and HL39650 and by The American Lung Association.
Exposure to hyperoxia affects metabolism and airway content of the major surfactant-associated protein and DSPC. However, specific effects vary depending on animal species, age, and level and duration of inspired oxygen. Young et al. (7) found increased lavage and lung DSPC content in adult rats exposed to $85 \%$ oxygen for $7 \mathrm{~d}$. Nogee and Wispé (8) reported increased airway content of DSPC in adult rats exposed to $85 \%$ oxygen for 3 or 7 d. This increase was associated with increased synthesis and secretion of the major surfactant-associated protein SP-A. In contrast, Ward and Roberts (9) reported decreased synthesis and secretion of DSPC by lung slices obtained from newborn rabbits exposed to $95 \%$ oxygen for $48 \mathrm{~h}$. Thus, hyperoxia causes a significant perturbation of surfactant homeostasis. As ATP is a potent stimulus for surfactant phospholipid secretion in vitro (5, 6) and hyperoxia has also been reported to modulate surfactant phospholipid secretion $(7,8)$, we tested whether changes in bronchoalveolar lavage ATP content are associated with hyperoxia-induced changes in DSPC secretion by measuring bronchoalveolar lavage ATP and disaturated phosphatidylcholine content in rats exposed to varying inspired oxygen concentrations.

\section{MATERIALS AND METHODS}

Materials. Male rats (150-250 g) were obtained from Charles River Breeding Laboratories, Inc., Wilmington, MA. ATP, ADP, AMP (all as sodium salts), adenosine, NADP, glucose-6-phosphate dehydrogenase, hexokinase, and tetrabutyl ammonium hydrogen sulfate were obtained from Sigma Chemical Co., St. Louis, MO.

Methods. Adult male Sprague-Dawley rats were exposed to hyperoxia or room air for the dose-response study. The average wt of the study animals was $350 \mathrm{~g}$ for the dose-response study, and $250 \mathrm{~g}$ for the time course. The oxygen-treated rats were housed in 3-cubic-foot Lucite chambers. Each chamber received oxygen at 6 liters/min, and oxygen concentration was measured continuously with an oxygen analyzer (Ventronics, Temecula, $\mathrm{CA}$ ). During the exposure, $\mathrm{CO}_{2}$ concentration in the chamber was less than $1 \%$. Room air control animals were maintained in open cages. Animals were allowed food and water ad libitum.

After the exposure period, animals received a lethal intraperitoneal injection of pentobarbital $(50 \mathrm{mg} / \mathrm{kg})$. The thorax was left undisturbed, and the tracheae were cannulated and connected to a U-tube water manometer. The lungs were slowly inflated to 30 $\mathrm{cm} \mathrm{H}_{2} \mathrm{O}$ pressure, and this vol was recorded as $\mathrm{V}_{30}$. The lungs were lavaged in situ 10 times with cold $\mathrm{PBS}, \mathrm{pH}=7.4$, containing 3.4-mM EDTA using a vol equal to $80 \%$ of $V_{30}$. Fresh buffer was used for each lavage. Total recovered lavage fluid from each animal had to be at least $90 \%$ of vol instilled to qualify for inclusion in the analysis, and there were no differences in lavage vol recovered from control versus oxygen-exposed rats. Pulmonary lavage was processed by centrifugation at $500 \times g$ for 10 min at $4^{\circ} \mathrm{C}$ to remove cell debris. The supernatants were then centrifuged at $12500 \times g$ for $10 \mathrm{~min}$ at $4^{\circ} \mathrm{C}$, and aliquots of the 
resultant supernatant were taken for determination of lactate dehydrogenase activity, ATP, and metabolite measurement.

To determine recovery of ATP using this methodology, tracer amounts of adenosine $5^{\prime}-O-\left(3-\left[{ }^{35} \mathrm{~S}-\right]\right.$ thiotriphosphate $)$ were added to the lungs of control animals which were then lavaged as above and not ventilated. Under these conditions, $98 \pm 6 \%$ of the instilled label was recovered by lavage (mean $\pm \mathrm{SEM}, n=$ 3 ). None of the control or oxygen-treated animals expired before being killed for the studies.

ATP and metabolite determination. ATP content was determined using a spectrofluorometric assay (10). Assay buffer contained 50-mM HEPES, 10-mM magnesium chloride, 5-mM glucose and 5-mM EDTA, $\mathrm{pH}=7.4$. In addition, the buffer also contained nicotinamide adenine dinucleotide phosphate $(50 \mu \mathrm{g})$, glucose-6-phosphate dehydrogenase (EC 1.1.1.49, 0.1 U) and hexokinase (EC 2.7.1.1, 1 U). The amount of ATP present in lavage fluid was determined by comparison to standard curves, and by utilizing a known amount of ATP as an internal standard.

ATP levels were also measured by ion-pair reversed-phase HPLC (11). Buffer A contained 0.1-M $\mathrm{KH}_{2} \mathrm{PO}_{4}, \mathrm{pH}=6$, with $8-\mathrm{mM}$ tetrabutyl ammonium hydrogen sulfate, and buffer $\mathrm{B}$ was buffer $A$ containing methanol $(60: 40, \mathrm{vol} / \mathrm{vol})$. The amount of ATP present in lavage fluid was determined by comparison to standards eluted by high performance liquid chromatography using the previously described method. ADP, AMP and adenosine levels were determined in a similar fashion. Results obtained with the spectrofluorometric assay and by HPLC were similar with $r=0.83$.

DSPC determinations. DSPC concentration of bronchoalveolar lavage fluid was determined as previously described (12).

Lactate dehydrogenase measurements. Lactate dehydrogenase levels were determined on lavage fluid utilizing previously described methods (5).

Statistical evaluation. ANOVA with Newman Keuls test was used to assess statistical significance where noted; $p<0.05$ was considered statistically significant.

\section{RESULTS}

ATP was uniformly detected in bronchoalveolar fluid from all animals. Bronchoalveolar lavage from control rats exposed to room air contained $155 \pm 12 \mathrm{nmol}$ of ATP (Table 1). There was a dose-dependent decrease in airway ATP content after oxygen exposure which reached $34 \pm 5 \mathrm{nmol}$ in bronchoalveolar lavage after exposure to $85 \%$ oxygen for $72 \mathrm{~h}$. The decrease in bronchoalveolar lavage ATP content was accompanied by a decrease in bronchoalveolar lavage content of ATP metabolites in a similar fashion, although adenosine content in bronchoalveolar lavage fluid was not significantly different after oxygen exposure (Table 1).

Although ATP and ATP metabolite content decreased in a dose-dependent fashion with oxygen exposure, disaturated phos-

Table 1. Dose-dependent effect of oxygen exposure on bronchoalveolar lavage content of ATP and ATP metabolites*

\begin{tabular}{lcccc}
\hline & \multicolumn{4}{c}{$\begin{array}{c}\mathrm{Fi}_{\mathrm{O}_{2}} \\
\text { (nmol/lavage) }\end{array}$} \\
\cline { 2 - 5 } & 0.21 & 0.75 & 0.80 & 0.85 \\
\hline ATP & $155 \pm 12$ & $146 \pm 37$ & $66 \pm 11 \dagger$ & $34 \pm 5 \dagger$ \\
ADP & $51 \pm 7$ & ND $\$$ & $35 \pm 8$ & $19 \pm 2 \dagger$ \\
AMP & $39 \pm 5$ & ND $\ddagger$ & $38 \pm 12$ & $20 \pm 2 \dagger$ \\
Adenosine & $13 \pm 4$ & ND $\$$ & $17 \pm 6$ & $11 \pm 1$ \\
\hline
\end{tabular}

* Rats were exposed to oxygen for $72 \mathrm{~h}$ and lavaged as described in "Materials and Methods." Results represent mean \pm SEM for 17 (control), four (0.75), $21(0.80)$ or $17(0.85)$ animals. ATP content was determined by the spectrofluorometric assay.

$\dagger p<0.05$ compared to control.

$\ddagger \mathrm{ND}=$ not determined.

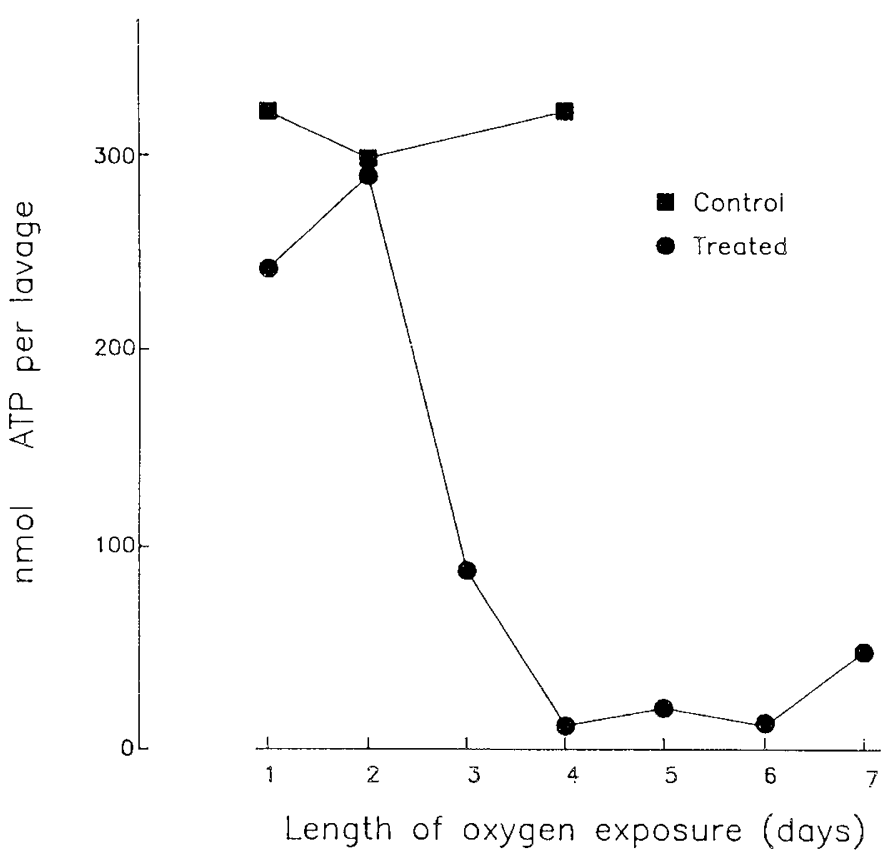

Fig. 1. Effect of hyperoxia on bronchoalveolar lavage ATP content varies with time of exposure. Rats were exposed to $85 \%$ oxygen (treated) or $21 \%$ oxygen (control) for the indicated times and lavaged as described in "Materials and Methods." Each point represents the average ATP content from bronchoalveolar lavage of two animals at each time point.

phatidylcholine concentrations increased. Bronchoalveolar lavage from control animals contained $130 \pm 20 \mu \mathrm{g}$ DSPC/lavage whereas lavage from animals exposed to $85 \%$ oxygen contained $1160 \pm 200 \mu \mathrm{g}$ DSPC/lavage (mean \pm SEM, $n=12$ )

In animals exposed to $85 \%$ oxygen, there was also a timedependent decrease in airway ATP content (Fig. 1). Airway ATP content decreased from $236 \mathrm{nmol} /$ lavage and $292 \mathrm{nmol} /$ lavage on $\mathrm{d} 1$ and 2 of oxygen exposure to a level of $18 \mathrm{nmol} /$ lavage in rats exposed to $85 \%$ oxygen for $4 \mathrm{~d}$. This low level of lavage ATP persisted for $7 \mathrm{~d}$ of oxygen exposure (Fig. 1).

Lactate dehydrogenase content of the bronchoalveolar lavage was measured as described in "Materials and Methods" and expressed relative to lactate dehydrogenase activity of bronchoalveolar lavage fluid from control rats $(100 \pm 30 \%, n=16)$. Bronchoalveolar lavage fluid from rats exposed to $80 \%$ oxygen contained $240 \pm 40 \%$ lactate dehydrogenase activity, whereas lavage from rats exposed to $85 \%$ oxygen contained $120 \pm 30 \%$ lactate dehydrogenase activity compared to controls (values are mean \pm SEM for 17-21 animals). The values obtained for oxygen-treated rats were not statistically different from the lactate dehydrogenase activity obtained by bronchoalveolar lavage of control animals. The vol of lavage fluid recovered relative to the vol of fluid instilled was at least $90 \%$ and was similar in control and treated animals.

\section{DISCUSSION}

After oxygen exposure, airway content of ATP and ATP metabolites fell in a time- and dose-dependent fashion. Although ATP is a potent stimulus for surfactant secretion in vitro $(5,6)$, whether ATP plays a similar role in vivo is not known. From the present data, ATP is present in the airway at concentrations sufficient to cause secretion of surfactant from type II cells. Assuming a vol of $100 \mu \mathrm{L}$ for the liquid lining the alveolar surface of the rat (13), the concentration of ATP would be on the order of $1 \mathrm{mM}$, which is in excess of the $\mathrm{EC}_{50}$ for surfactant phospholipid secretion in vitro $\left(\sim 1 \times 10^{-6} \mathrm{M}\right.$, Ref. 5$)$. However, DSPC levels increased while ATP concentrations fell in lavage 
from oxygen-treated animals, arguing against regulation of oxygen-induced DSPC secretion by ATP.

The source of ATP that could activate type II cell purinoceptors in vivo is presently unclear. ATP is released from vascular endothelial cells and from smooth muscle cells in culture (14). This release of ATP appears to be selective as it is not accompanied by concomitant lactate dehydrogenase release. As lung contains both endothelial cells and smooth muscle cells, it is possible these cell types release ATP in vivo to regulate surfactant secretion in response to other exogenous stimuli. Damage to alveolar lining cells also may result in release of cytosolic ATP into the airway, as the normal intracellular ATP concentration is 4-5 mM in healthy cells. However, in the event of cell damage, ATP levels would be expected to fall before ultimate demise of the cell, making this a less likely source of ATP in the airway in vivo. It has also been previously noted, using the same adult rat model, that airway lacks intracellular precursor forms of the major surfactant apoprotein (8). Therefore, damage to type II cells does not account for the present observations, although damage to other alveolar lining cells is possible. Nonadrenergic, noncholinergic nerve endings which utilize ATP as a neurotransmitter have been identified in guinea pig vas deferens (15) and gall bladder (16). Such nerve endings may also exist in lung and release ATP in response to various exogenous stimuli, such as hyperventilation.

The fate of bronchoalveolar ATP is also unclear. The presence of ecto-ATPases has been reported on endothelial cells from pig aorta as well as rat hepatocytes and human B lymphocytes (1719). If ecto-ATPases are present on alveolar lining cells, they would provide a mechanism for down regulation of surfactant secretion stimulated by ATP. Such ecto-ATPases may also be activated during oxygen exposure of the animals, leading to the decreased levels of ATP we observed in oxygen-treated animals. However, levels of ATP metabolites were similarly decreased, although adenosine could be taken up by alveolar lining cells via facilitated diffusion if the ATP, ADP, and AMP were ultimately degraded to adenosine. Ecto-kinases have also been reported on the surface of neural cells and spermatozoa $(20,21)$. Such kinases, if present in the alveolar lining, could degrade ATP to ADP. However, ADP and AMP would also have to be degraded by ecto-nucleotidases to adenosine which is then taken up by lining cells to explain results of the present study. Alveolar lining cells may also transport ATP and ATP metabolites into the cytosol. However, ATP is a charged molecule at physiologic $\mathrm{pH}$, and no specific transport system for ATP is known to exist in the alveolus.

ATP may be degraded in bronchoalveolar lavage fluid from oxygen-treated animals more readily than ATP in bronchoalveolar lavage fluid from control animals. Although lactate dehydrogenase content was similar in lavage from treated and control animals, it is possible that cells present in bronchoalveolar lavage from oxygen-treated animals take up ATP more readily or degrade ATP more rapidly than cells in control rats. This does not appear likely as concentrations of ATP metabolites fell in concert with ATP concentrations. Altered recovery of ATP in bronchoalveolar lavage from treated animals is also not a sufficient expla- nation for the alterations in ATP concentrations we observed. More than $90 \%$ of instilled lavage fluid was recovered in both treated and control animals. We also recovered $98 \%$ of radiolabeled ATP which was instilled in control animals.

In summary, this is the first demonstration of measured airway ATP levels in vivo and indicates presence of ATP in bronchoalveolar lavage fluid at levels which modulate surfactant phospholipid secretion from isolated alveolar type II cells in vitro. There is also a significant decrease in airway ATP content with exposure to oxygen, which may result in altered surfactant homeostasis and impaired lung function observed in similar clinical situations.

\section{REFERENCES}

1. Dobbs LG, Mason RJ 1978 Pulmonary alveolar type II cells isolated from rat. $\mathrm{J}$ Clin Invest 63:378-387

2. Dobbs LG, Mason RJ 1978 Stimulation of secretion of disaturated phosphatidylcholine from isolated alveolar type II cells by 12-O-tetradecanoyl-13phorbol acetate. Am Rev Respir Dis 118:705-713

3. Marino PA, Rooney SA 1980 Surfactant secretion in the newborn rabbit lung slice model. Biochim Biophys Acta 620:509-519

4. Rice WR, Osterhoudt KC, Whitsett JA 1984 Effect of cytochalasins on surfactant release from alveolar type II cells. Biochim Biophys Acta 805:12-18

5. Rice WR, Singleton FM $1987 \mathrm{P}_{2 y}$-purinoceptor regulation of surfactant secretion from rat isolated alveolar type II cells is associated with mobilization of intracellular calcium. Br J Pharmacol 91:833-838

6. Gilfillan AM, Rooney SA 1987 Purinoceptor agonists stimulate phosphatidylcholine secretion in primary cultures of adult rat type II pneumocytes. Biochim Biophys Acta 917:18-23

7. Young SL, Crapo JD, Kremers SA, Brumley GW 1982 Pulmonary surfactant lipid production in oxygen-exposed rat lungs. Lab Invest 46:570-576

8. Nogee LM, Wispe JR 1998 Effects of pulmonary oxygen injury on airway content of surfactant-associated protein A (SP-A). Pediatr Res 24:568-573

9. Ward JA, Roberts RJ 1984 Effect of hyperoxyia on phosphatidylcholine synthesis, secretion, uptake and stability in the newborn rabbit lung. Biochim Biophys Acta 796:42-50

10. Williamson JR, Corkey BE 1969 Adenine nucleotides. Methods Enzymol 13:488-491

11. Stocchi V, Cucchiarini L, Canestrari F, Piacentini MP, Fornaini G 1987 A very fast ion-pair reversed-phase HPLC method for the separation of the most significant nucleotides and their degradation products in human red blood cells. Anal Biochem 167:181-190

12. Mason RJ, Nellenbogen J, Clements JA 1976 Isolation of disaturated phosphatidylcholine with osmium tetroxide. J Lipid Res 17:281-294

13. Weibel ER, Untersee P, Gil J, Zulauf M 1973 Morphometric estimation of pulmonary diffusion capacity VI: effect of varying positive pressure inflation of air spaces. Respir Physiol 18:285-308

14. Peterson JD, Gordon JL 1979 Vascular endothelial and smooth muscle cells in culture selectively release adenine nucleotides. Nature 281:384-386

15. Lew MJ, White TD 1987 Release of endogenous ATP during sympathic nerve stimulation. Br J Pharmacol 92:349-355

16. Takahashi T, Kusunoki M, Ishikawa Y, Kantoh M, Yamamura T, Utsunomiya J 1987 Adenosine 5'-triphosphate release evoked by electrical nerve stimulation from the guinea-pig gall bladder. Eur J Pharmacol 134:77-82

17. Gordon EL, Pearson JD, Slakey LL 1986 The hydrolysis of extracellular adenine nucleotides by cultured endothelial cells from pig aorta. J Biol Chem 261:15496-15504

18. Haussinger D, Stehle T, Gerok W, Tran-Thi T, Decker K 1987 Hepatocyte heterogeneity in response to extracellular ATP. Eur J Biochem 169:645-650

19. Brankiewicz J, Dosch H, Cohen A 1988 The extracellular nucleotide metabolism in human B and T lymphocytes. J Biol Chem 263:7094-7098

20. Erhlich YH, Davis TB, Bock E, Karonecki E, Lennox RH 1986 Ecto-protein kinase activity on the external surface of neural cells. Nature 320:67-70

21. Haldar S, Majumder GC 1986 Phosphorylation of external cell-surface proteins by an endogenous ecto-protein kinase of goat epididymal intact spermatozoa. Biochim Biophys Acta 887:291-303 